Monatsschr Kinderheilkd 2014 [Suppl 2]

162:105-115

DOI 10.1007/s00112-014-3210-x

๑) Springer-Verlag Berlin Heidelberg 2014

\section{Abstracts 2014}

\section{Gemeinsame Jahrestagung der DGKJ, DGSPJ, DGKCH, des BeKD, der DGPI, GKJR, GPOH und der AG Pädiatrische Radiologie der DRG}

\author{
11. bis 14. September 2014, Leipzig
}

\section{Wissenschaftliche Leitung}

Prof. Dr. med. James Beck, Tagungspräsident DGKJ

Dr. med. Carsten Wurst, Tagungspräsident DGSPJ

Prof. Dr. med. Guido Fitze, Tagungspräsident DGKCH

Frauke Leupold, Tagungspräsidentin BeKD

Prof. Dr. med. Michael Borte, Tagungspräsident GKJR

PD Dr. med. Roswitha Bruns, Tagungspräsidentin DGPI

Prof. Dr. med. Angelika Eggert, Tagungspräsidentin GPOH

Prof. Dr. med. Hans-J. Mentzel, Tagungspräsident

AG Pädiatrische Radiologie DRG

\section{Interdisziplinäre Sitzungen}

\author{
Erbrechen
}

INTDIS-SY-ER-2

Bildgebung bei Erbrechen

Stenzel $\mathrm{M}^{1}$, Mentzel $\mathrm{HJ}^{\prime}$

IInstitut für Diagnostische und Interventionelle Radiologie, Sektion Pädiatrische Radiologie, Universitätsklinikum der Friedrich-Schiller-Universität Jena, Jena

Wiederholtes Erbrechen und Nüchternerbrechen sind bis zum Beweis des Gegenteils Ausdruck einer schweren zugrunde liegenden Erkrankung. In Abhängigkeit von weiteren Auffälligkeiten in der klinischen und paraklinischen Untersuchung stellt die Bildgebung ein Mittel zur Verfügung, vermutete Diagnosen zu bestätigen oder widerlegen. Bei pathologischen Prozessen des Gastrointestinaltrakts stellt die Sonographie des Abdomens die Methode der ersten Wahl dar. Mit ihr gelingt es unter anderem zuverlässig, die hypertrophe Pylorusstenose zu diagnostizieren. Erbrechen kann jedoch auch zentral bedingt sein. Mit den bildgebenden Methoden lässt sich eine Liquorpassagestörung, wie sie bei Hirntumoren zu finden ist, nachweisen. Die Schnittbildgebung dient neben dem Nachweis auch der Ausschlussdiagnostik.

\section{INTDIS-SY-ER-3}

Konservativ-pädiatrische Ursachen von Erbrechen

Kurzai $M$.

'Universitätsklinikum Jena, Klinik für Kinder- und Jugendmedizin, Jena

Erbrechen ist die durch reflektorische Mechanismen gesteuerte propulsive Entleerung des Mageninhalts entgegen der natürlichen Richtung. Es muss begrifflich von der Regurgitation abgegrenzt werden, bei der das Zurückfließen des Speisebreis in die Mundhöhle nicht aktiv stattfindet. Erbrechen ist ein häufiges Symptom und meist Ausdruck einer harmlosen, selbstlimitierten Störung. Es kann aber auch Symptom einer lebensbedrohlichen Erkrankung sein. Das Alter des Patienten, die gezielte Befragung hinsichtlich eventueller Begleitsymptome und eine sorgfältige klinische und neurologische Untersuchung liefern wichtige Hinweise für die Eingrenzung der breiten Differenzialdiagnose. Diese umfasst entzündlich-infektiöse, medikamentös-toxische, vegetative, neurologische, metabolische, endokrinologische und allergische Ursachen. Galliges Erbrechen sollte immer als Warnsignal für mögliche bedrohliche Situationen wie intestinale Obstruktion oder Ischämie wahrgenommen werden, die eventuell ein rasches operatives Vorgehen erfordern. Bei wiederkehrendem Auftreten ohne organischen Befund kommen eine Essstörung und das Syndrom des zyklischen Erbrechens in Betracht. Anhand von Fallbeispielen soll in diesem Vortrag das Vorgehen bei der differenzialdiagnostischen Abklärung dargestellt werden. 


\section{Jahresrückblicke}

\section{INTDIS-SY-JR-3}

Behandlungskonzepte für die Sichelzellkrankheit im Wandel

Kunz J.'

'ZKJM, Heidelberg

Die Sichelzellkrankheit als Prototyp einer „molekularen Krankheit“ ist seit vielen Jahrzehnten pathophysiologisch und klinisch gut charakterisiert. Dennoch bleibt die Behandlung dieser chronischen Multisystemkrankheit eine Herausforderung.

2014 wurde die Leitlinie zur Behandlung der Sichelzellkrankheit grundlegend überarbeitet. Wesentliche Neuerung ist die erweiterte Indikation zur Gabe von Hydroxycarbamid, die sich unter anderem aus den Erkenntnissen der BABY-HUG-Studie ableitet. Diese randomisierte, placebokontrollierte Studie konnte zeigen, dass die Gabe von Hydroxycarbamid bei jungen Kindern mit Sichelzellkrankheit das Fortschreiten von Organschäden verlangsamen kann. Die zweite Neuerung ist die erweiterte Indikation zur allogenen Stammzelltransplantation, die sich aus den Erfahrungen geringer transplantationsbedingter Mortalität bei Verfügbarkeit eines HLA-identischen Stammzellspenders ableitet. Ein Register, das ab 2014 Patienten rekrutiert, soll die Häufigkeit der Komplikationen der Sichelzellkrankheit in Deutschland und den Erfolg der angewendeten Therapiemodalitäten dokumentieren.

In Deutschland wurden in den vergangenen zwei Jahren durch Neugeborenenscreeningprojekte erstmals belastbare Daten zur Prävalenz der Sichelzellkrankheit erhoben. Die Frequenz Neugeborener mit Sichelzellkrankheit lag im Bereich von 1/250o für Berlin und Hamburg, deutlich niedriger für den Südwesten Deutschlands. Die durch Screening-Pilotprojekte und Register gewonnenen Daten sollen als Argumentationsgrundlage für die Einführung eines generellen Neugeborenenscreenings, wie es in zahlreichen europäischen Nachbarstaaten seit Jahren etabliert ist, in Deutschland dienen und so die Versorgung dieser wachsenden Patientengruppe verbessern.

\section{Transition 1 - Bridging the gap}

\section{INTDIS-SY-TB-1}

\section{Lost in Transition - erfolgreich therapiert und doch verloren?}

Stier B.J.M. ${ }^{1}$

'Kinder- und Jugendarztpraxis, Butzbach

Transition ist - anders als Transfer - ein Prozess, der sich über einen längeren Zeitraum hinzieht. Viel zu häufig finden sich Jugendliche in Betreuungsstrukturen, die dem Sinn der Transition zuwiderlaufen, zwischen zwei Welten oder treffender: zwischen den Stühlen. Nach beeindruckenden therapeutischen Erfolgen in der pädiatrischen Behandlung und Kontrolle chronischer Erkrankungen droht - meist plötzlich - eine medizinische Unterversorgung durch mangelnde Koordination des Prozesses. Etwa 15\% aller Kinder in Deutschland leiden an einer chronischen Erkrankung. Fast jede vierte Familie lebt mit mindestens einem chronisch erkrankten Kind. Diese Kinder werden erwachsen und können dann nicht mehr vom Pädiater betreut werden. Obwohl schon seit einigen Jahren im Fokus, ist Transition immer noch zu wenig institutionalisiert, standardisiert und strukturiert. Nach wie vor zeigt die „Transitionslandschaft" überwiegend „weiße Flecken“. Die resultierende Unterversorgung äußert sich z. B. durch eine Zunahme der Therapieabbrüche und/oder mangelnde Medikamenteneinnahme. Dabei bedeutet Transition auch, den Übergang von ganzheitlicher und familienorientierter Versorgung $\mathrm{zu}$ eher organspezifisch und personenbezogener Betreuung zu meistern. Der trianguläre Behandlungsstil des Kindesalters (Kind-Eltern-Arzt) muss, gerade bei chronisch erkrankten Jugendlichen, dem bipolaren weichen. Erfolgreiche Transition gelingt nur als gemeinschaftliche Aufgabe aller pädiatrischer Fachbereiche in Zusammenarbeit mit den Leistungsträgern. Sie basiert auf einer strukturellen, personellen und nicht zuletzt finanziellen Förderung von jugendgerechten Versorgungsstrukturen im niedergelassenen und Klinikbereich. Nur gelingende Transition verhilft dem therapeutischen Fortschritt zu bleibendem Erfolg.

\section{INTDIS-SY-TB-2}

\section{Erwachsenwerden mit chronischen Erkrankungen}

\section{Siegmund B.}

'Charité-Universitätsmedizin Berlin, CBF, Med. Klinik m. S. Gastroenterologie, Rheumatologie, Infektiologie, Berlin

Als Transition wird der geplante Wechsel chronisch-kranker Jugendlicher und junger Erwachsener von der kinderärztlichen Versorgung in die Erwachsenenmedizin bezeichnet. Die Transition fällt in der Regel in die Lebensphase der späten Adoleszenz, d. h. in einen Zeitraum mit hoher Vulnerabilität. Die Folge sind Behandlungsabbrüche oder -unterbrechungen mit Auswirkungen auf den Krankheitsverlauf und sozioökonomischen Konsequenzen. Bereits 2009 wurde von Sachverständigenrat die Betreuung chronisch kranker Jugendlicher in der Übergangsphase als mangelhaft bewertet.

Mit dem Ziel die Ist-Situation zu evaluieren sowie einen Transitionsmodell zu erarbeiten, wurde 2012 die Task Force Transition durch die Deutsche Gesellschaft für Innere Medizin und die Deutsche Gesellschaft für Kinder- und Jugendmedizin gegründet. Im Mittelpunkt der Arbeitsgruppe für ein Transitionsmodell stehen Jugendliche, die im Erwachsenenalter die Autonomie erlangen. Nach Durchsicht verschiedener Modelle wurde das Berliner Transitionsprogramm, das von den DRK-Kliniken zunächst für die Bereiche Epilepsie und Diabetes erarbeitet wurde, ausgewählt. Innerhalb dieses Modellprogramms wurden fächerübergreifende Strukturen wie Transitionsheft, Epikrise, Fallmanagement, krankheitsspezifische Materialen und die Vergütung etabliert. Das Modellprogramm wurde mittlerweile auf andere Erkrankungen wie Rheumatologie, chronisch entzündliche Darmerkrankungen, Nephrologie und Neurologie sowie auch räumlich auf andere Bundesländer ausgedehnt werden. Die Mitglieder der Task Force sind sich einig, dass nur durch ein einheitliches Konzept, dass durch beide Fachgesellschaften getragen wird, die strukturellen Defizite aber auch die assoziierten finanziellen Probleme gelöst werden können.

\section{INTDIS-SY-TB-3}

"Erwachsenwerden mit Rheuma“ - als Modell für die Transition bei chronischen Krankheiten

\section{Minden $K^{1,2}$}

${ }^{1}$ Charite Universitätsmedizin Berlin, Kinderklinik, Rheumatologie, Berlin, ${ }^{2}$ Deutsches Rheuma-Forschungszentrum Berlin, Programmbereich Epidemiologie, Berlin

Gelenkrheuma ist eine der häufigsten chronischen Krankheiten im Kindes- und Jugendalter; etwa jedes tausendste Kind ist hiervon betroffen. Bei mindestens jedem 2. Patienten bleibt die Erkrankung bis ins Erwachsenenalter aktiv bzw. behandlungsbedürftig. Die Betroffenen sind deshalb - wie andere chronisch kranke Jugendliche auch - in der kritischen Übergangsphase vom Kindes- ins Erwachsenenalter doppelt belastet: einerseits kann sich das Rheuma auf die normale psychosoziale und körperliche Entwicklung auswirken, andererseits kann sich altersspezifisches Risikoverhalten dauerhaft ungünstig auf den Krankheitsverlauf auswirken. So konsumiert z. B. jeder 5. junge Rheumatiker Nikotin oder mindestens 1-mal pro Woche Alkohol, ca. jeder 10. Drogen. Damit unterscheiden sich rheumakranke Jugendliche nicht von ihren Alterskameraden. Für sie birgt dieses Verhalten aber höhere Risiken. Bei der Versorgung junger Rheumatiker gilt es insofern nicht nur krankheitsspezifische Aspekte, sondern auch entwicklungstypische Besonderheiten zu berücksichtigen. 
Das Ziel in der Behandlung Rheumakranker besteht, solange Heilung nicht gelingt, in der Sicherstellung einer lebenslang bestmöglichen Lebensqualität. Das erfordert den Patienten in der wegbereitenden Phase der Adoleszenz seinen individuellen medizinischen, psychosozialen und Bildungs-/beruflichen Bedürfnissen entsprechend zu unterstützen und seine kompetente medizinische Versorgung im Erwachsenenalter zu organisieren. Hierfür sind über die Regelversorgung hinausgehende spezielle Versorgungsleistungen vonnöten. Verschiedene Maßnahmen und Programme wurden bereits zur Verbesserung der Versorgung junger Rheumatiker implementiert, dennoch bestehen nach wie vor erhebliche Versorgungsdefizite. Diese sind nicht auf die Rheumatologie beschränkt.

\section{Arzneimittelsicherheit}

\section{INTDIS-SY-AM-1}

Arzneimittelsicherheit im Kindesalter - zu Risiken und Nebenwirkungen

\section{Boos J.' \\ 'Universitätsklinikum Münster, Pädiatrische Hämatologie und Onkologie, Münster}

Kinder haben sich in Bezug auf unerwünschte Arzneimitteleffekte als besondere Risikogruppe erwiesen. Intensive Diskussionen über den kritischen hohen Bedarf an Medikamenten außerhalb des Zulassungsrahmens führten aber zu einer ganzen Reihe von gesetzlichen Änderungen, die letztlich eine bessere und damit sicherere Versorgung von Kindern mit Arzneimittel zum Ziel hatten. Zeitgleich entwickeln sich aber auch arzneiliche Innovationen und Arzneimittelzulassungen sehr dynamisch. Die Hoffnung für einen Patienten ist dann oft einer nicht wirklich bekannten Sicherheitslage entgegenzusetzen.

Selbst bei altbekannten Medikamenten können Arzneimittelrisiken nie vollständig bekannt und vorhersehbar sein, weil ständig neue individuelle Therapiesituationen und -kombinationen entstehen. Risiken leiten sich dabei aus den Wirkstoffen, den Hilfsmitten, den Darreichungsformen und Applikationsroutinen und ggf. auch Pädiatrie-typischen Komedikationen ab.

In der Kinder- und Jugendmedizin werden systematische Sicherheitsstudien das Problem nur sehr begrenzt lösen. Mit den neuen arzneimittelgesetzlichen Regelungen wurden aber auch und gerade für Kinder adaptierte Möglichkeiten geschaffen, um neu auftretende Arzneimittelnebenwirkungen möglichst frühzeitig zu erkennen und bekannt $\mathrm{zu}$ machen. Eine umfassende Beteiligung an den Meldesystemen für beobachtete Arzneimittelnebenwirkungen ist daher im Sinne der Patienten wichtig.

Im Vortrag werden Hintergründe und Kernelemente der neuen Gesetzgebung zur Pharmakovigilanz sowie die Meldewege und Informationsquellen vorgestellt.

\section{INTDIS-SY-AM-2}

Wie sicher sind die "First-line-Therapeutika" der juvenilen idiopathischen Arthritis in der Langzeitanwendung?

\section{Michels $H^{?}$}

'Deutsches Zentrum für Kinder- und Jugendrheumatologie, Garmisch-Partenkirchen

Als „First-line-Therapeutika“ sollen hier nichtsteroidale Antirheumatika (NSAR), Glucocorticoide (GC) und Methotrexat (MTX) angesprochen werden.

NSAR werden von Kindern besser vertragen als von Erwachsenen. Relevante Probleme bei Langzeitanwendung sind selten und beziehen sich insbesondere auf gastrointestinale (Ulcus, Blutung), hepatotoxische (Transaminasen), renale (Papillennekrose, Nephritis, nephrotisches
Syndrom) und zentralnervöse (Konzentrationsstörungen häufiger) unerwünschte Wirkungen (UEW). Kardiovaskuläre UEW wie Myokardinfarkt oder zerebrale Ischämie scheinen bei Kindern extrem selten zu sein, verwertbare Daten fehlen.

Die von GC-Langzeittherapien hinlänglich bekannten UEW wie Kleinwuchs, Cushing-Habitus oder Osteoporose haben dazu geführt, dass GC bei Kindern und Jugendlichen nicht mehr für eine systemische antiarthritische Langzeittherapie verwendet werden. Im Vordergrund stehen stattdessen die intraartikuläre (relevante Gewebsatrophien in ca. 1\%) und ophthalmologische Lokaltherapie (Glaukom, GC-Katarakt). Gelegentlich werden „Überbrückungstherapien“ mit Methylprednisolon-Infusionen („Pulstherapie“) durchgeführt, die bei prolongierter Anwendung UEW wie Hypertonie oder Hyperglykämie verursachen können.

MTX wird bei der Behandlung der JIA als Immunsuppressivum mit dem günstigsten Verhältnis „Effektivität/UEW“ angesehen. Mindestens die Hälfte der Patienten entwickeln jedoch Abneigung und Ekel gegen MTX, was häufig zur Dosisminderung oder Therapiebeendigung führt. Die als besonders relevant anzusehenden Gefahren - Entwicklung einer Leberzirrhose, eines Malignomes und einer MTX-Pneumonitis - scheinen sehr selten zu sein.

\section{Infektiologische Notfälle}

\section{INTDIS-SY-IN-1}

Symposium Infektiologische Notfälle

\section{Prelog M.}

'Universität Würzburg, Universitäts-Kinderklinik, Würzburg

Zu den systemischen inflammatorischen Erkrankungen zählen im weiteren Sinne Kollagenosen, Vaskulitiden und periodische Fiebersyndrome. Periodische Fiebersyndrome sind autoinflammatorische Zustandsbilder, die durch episodisch auftretendes oder kontinuierliches Fieber und spezifische klinische Präsentationen (z. B. Gelenksbeteiligung, Hautsymptome, Serositiden, gastrointestinale Symptome, Augen- und ZNS-Beteiligung) gekennzeichnet und meist hereditär sind. Dazu gehören das Familiäre Mittelmeerfieber, Cryopyrin-assoziierte periodische Fiebersyndrome, wie das Familiäre Kälte-asssoziierte Syndrom (FACS), das Muckle-Wells-Syndrom (MWS) und das CINCA-Syndrom als schwerste Ausprägung, das TNF-Rezeptor-assoziierte periodische Syndrom (TRAPS), das Hyper-IgD-Syndrom und periodisches Fieber mit aphthöser Stomatitis, Pharyngitis und Lymphadentitis (PFAPA). Auch die systemische Juvenile Idiopathisch Arthritis (sJIA) wird auf Grund des inflammatorischen Profils als autoinflammatorische Erkrankung verstanden, wobei es im akuten Schub zu lebensbedrohlichen Situationen (z. B. durch Perikarderguss) kommen kann. Als Komplikation ist das Makrophagen-Aktivierungssyndrom gefürchtet. Inflammatorische Erkrankungen können zu schweren destruktiven Verläufen mit Organbeteiligung oder zur Organinsuffizienz durch Amyloidose führen. Eine frühzeitige Diagnose der Erkrankung und eine effiziente anti-inflammatorische Therapie sind essentiell für den Langzeitverlauf und Prognose.

\section{INTDIS-SY-IN-4}

\section{Gibt es eine aktuelle Sepsistherapie für das Kind?}

\section{Bosk A. ', Pöschl J. ${ }^{2}$, Simon A. ${ }^{3}$, Wendt C. ${ }^{4}$, Hufnagel M. ${ }^{5}$}

'Diakonissen-Stiftungs-Krankenhaus, Pädiatrie, Speyer, ${ }^{2}$ Universitätsklinik, Neonatologie und Pädiatrische Intensivmedizin, Heidelberg, ${ }^{3}$ Universitätsklinik, Pädiatrische Onkologie und Hämatologie, Homburg/Saar, ${ }^{4}$ Labor Limbach, Heidelberg, ${ }^{5}$ Universitätsklinik, Allgemeinpädiatrie, Neonatologie, Infektiologie, Freiburg

Sepsis stellt immer noch einen hohen Morbiditäts- und Mortalitätsfaktor auch im Kindesalter dar. Es existieren verschiedene Therapie- 
Grundsätze und internationale Leitlinien dazu. Der Vortrag stellt die in Bearbeitung bzw. Diskussion befindlichen deutschen Leitlinien für die AWMF vor und fokussiert auf Definition (insbesondere auch zur Abbildung im DRG-System), Diagnose und Stellenwert der Diagnostik sowie auf Prävention und Therapie der Sepsis im Kindes- und Jugendalter jenseits der Neonatalperiode.

Unter anderem finden folgende Inhalte Berücksichtigung:

- Auswahl des zentralen Zugangswegs, ZVK-Pflege und -Wechsel,

- Surveillance-Programme,

- Impfungen,

- Antibiotika,

- Immunstimulation und -modulation,

- Immunglobuline,

- Glucocorticosteroide,

- rekombinantes aktiviertes Protein C und Protein-C-Konzentrat,

- Glucose-Kontrolle und Insulintherapie,

- „nutritional support“ und „immunonutrition“,

- Kreislauftherapie: Flüssigkeitsregime, Katecholamine, invasive und nichtinvasive Kreislaufüberwachung.

Dabei werden auch etliche Therapieansätze diskutiert, die die Evidenz ihres Stellenwerts bislang schuldig blieben.

\section{Transition 3 - Ergebnisse aus den Kommissionen}

\section{INTDIS-SY-TE-1}

AG Transition der DGKJ, DGIM, DGN - Aufgaben, Ziele und bisherige Ergebnisse

Rodeck B. ${ }^{1}$, Bösebeck F. ${ }^{2}$, Hansen G. ${ }^{3}$, Hauffa B. ${ }^{4}$, Müther S. ${ }^{5}$, Reincke M. ${ }^{6}$, Siegmund B. ${ }^{7}$, von Moers A. ${ }^{5}$

${ }^{1}$ Christliches Kinderhospital, Osnabrück, ${ }^{2}$ Diakoniekrankenhaus Rotenburg, Klinik für Neurologie, Rotenburg, ${ }^{3}$ Medizinische Hochschule Hannover, Päd. Pneumologie, Hannover, ${ }^{4}$ Universität Duisburg-Essen, Klinik für Kinderund Jugendmedizin, Essen, ${ }^{5}$ DRK Klinikum Westend, Klinik für Kinder- und Jugendmedizin, Berlin, 'LMU Klinikum der Universität München, Medizinische Klinik und Poliklinik IV, München, ${ }^{7}$ Charité Universitätsmedizin Berlin, Medizinische Klinik I, Berlin

Die Transition vom Jugendlichen- zum Erwachsenenalter in unserem Gesundheitssystem wurde bislang seitens des Gesetzgebers und der Kostenträger nicht strukturell adressiert. Es fehlt eine fächerübergreifende Struktur, die den Prozess der Transition nicht nur begleitet sondern auch vergütet. Die DGKJ und die Deutsche Gesellschaft für Innere Medizin (DGIM) haben daher 2012 die Arbeitsgruppe Transition gegründet. Anfang 2013 trat die Deutsche Gesellschaft für Neurologie (DGN) hinzu. Die AG Transition hat nach Überprüfung der im Konvent für fachliche Zusammenarbeit der DGKJ bekannten Transitionsprojekte das Berliner TransitionsProgramm (BTP) als förderungswürdig anerkannt, da es sich für einen indikationsübergreifenden und überregionalen Einsatz eignet. Die AG ist mit verschiedenen Entscheidungsträgern der Gesundheitspolitik in einen Dialog eingetreten, um die Struktur und die Finanzierungsmöglichkeiten (z. B. $\$ 43$ SGB V) eines einheitlichen Transitionskonzepts zu klären.

Das BTP kann bundesweit etabliert werden, braucht dazu aber eine auch bundesweit operierende Organisation. Der Bundesverband Bunter Kreis e. V. mit 78 Standorten in der Bundesrepublik Deutschland hat sich grundsätzlich bereit erklärt, sich mit dieser Aufgabe zu befassen und Fallmanagementstrukturen regional aufzubauen.

Entscheidend für die Zukunft einer kompetenten und effektiven Transitionsversorgung sind die Sicherstellung der kostendeckenden Finanzierung und die optimale Kooperation der beteiligten Fachdisziplinen aus Pädiatrie und der Erwachsenenmedizin. Durch eine gute Begleitung des Transitionsprozesses wird die Therapietreue der betroffenen Patienten gestärkt, so dass sich eine Investition der Kostenträger in diesen Bereich auch unter volkswirtschaftlichen Gesichtspunkten „lohnt“.
INTDIS-SY-TE-2

Das Berliner TransitionsProgramm. Ein Strukturkonzept für den Übergang chronisch kranker Jugendlicher in die Erwachsenenmedizin

\section{Müther S. ${ }^{1}$}

'DRK Kliniken Berlin | Westend, Berliner TransitionsProgramm, Berlin

In Deutschland ist die Transition generell nicht einheitlich geregelt. Ein geplanter Übergang in eine erwachsenenmedizinische Spezialbetreuung erfolgt nur für einzelne Krankheitsbilder auf der Basis lokaler Initiativen. Um die Transition in Deutschland flächendeckend und indikationsübergreifend zu regeln, bedarf es einer strukturierten Transition mit definierten Leistungen und einer Koordination des gesamten Prozesses durch ein Fallmanagement sowie einer geregelten Kostenübernahme. Das Berliner TransitionsProgramm (BTP) erfüllt diese Kriterien. Es ist an den DRK Kliniken Berlin angesiedelt, die als Managementgesellschaft fungieren und wird von der Robert Bosch Stiftung gefördert. Die DRK Kliniken schließen Verträge mit den Krankenkassen und mit kooperierenden ambulanten Zentren. Dies sind beispielsweise Vertragspartner in niedergelassenen Praxen bzw. Medizinischen Versorgungszentren, Spezialambulanzen oder Sozialpädiatrischen Zentren. Das Fallmanagement ist für alle Patienten des Programms unabhängig von den betreuenden Einrichtungen an den DRK Kliniken Berlin angesiedelt. Das inhaltliche Konzept wurde von einer Arbeitsgruppe an den DRK-Kliniken Berlin in Zusammenarbeit mit dem IGES Institut zunächst für die Krankheitsbilder Epilepsie und Diabetes mellitus Typ 1 entwickelt und in Berlin und Brandenburg über 2 Jahre erprobt (2008-2012). Seit letztem Jahr ist das Programm auch auf die Indikationen chronisch entzündliche Darmerkrankungen, juvenile rheumatische Arthritis, neuromuskuläre Erkrankungen und terminale Niereninsuffizienz ausgeweitet. Derzeit wird das Programm in Berlin, Brandenburg, Hamburg, Schleswig Holstein, Mecklenburg Vorpommern und Niedersachsen angeboten.

\section{Das Schädel-Hirn-Trauma im Kindesalter}

\section{INTDIS-SY-SH-4 \\ Ist ein leichtes SHT wirklich leicht? - Rationale Behandlung aus sozialpädiatrischer Perspektive}

John . $^{\prime}$

'SPZ f. chronisch kranke Kinder/ Charité Berlin, Berlin

Im Vortrag werden, unter Beachtung der aktuellen Datenlage zum Outcome nach leichtem SHT, des Qualitätspapiers zur Sozialpädiatrische Nachsorge von Kindern/Jugendlichen nach SHT (Dr. med. Christoph Kretzschmar Sozialpädiatrisches Zentrum am Städtischen Krankenhaus Dresden-Neustadt) sowie eigener klinischer Erfahrungen der SHT-Sprechstunde des SPZ der Charité, die Chancen eines sozialpädiatrischen Behandlungsangebots sowie die Rolle einer entwicklungsneuropsychologischen Perspektive, zur Gewährleistung einer rationalen Behandlung dieser Patentengruppe, dargestellt. 


\section{Transition 4 - Nephrologie, zystische Fibrose und Erwachsenenmedizin}

\section{INTDIS-SY-TP-1}

\section{Transition bei Patienten mit CF}

\author{
Smaczny $C^{1}$ \\ 'Universitätsklinik Frankfurt/Main, Goethe Universität, Pneumologie, \\ Christiane Herzog CF-Zentrum, Frankfurt am Main
}

Ein CF-Kranker muss sich während der Adoleszenz neben den üblichen Herausforderungen dieser Zeit auch mit noch vielseitigen Besonderheiten seiner Erkrankung auseinandersetzen. In dieser Zeit steht auch der Wechsel aus der Pädiatrie in die Erwachsenen Medizin (Transfer) an. Ein Transfer ohne Transition ist jedoch zum Scheitern verurteilt. Die Transition ist im Gegensatz zum Transfer kein Ereignis sondern ein Prozess und ist als Vorbereitungszeit auf eine gezielte Überführung adoleszenter Patienten aus pädiatrischen in erwachsenenorientierte Versorgungssysteme zu verstehen. Dieser Prozess verläuft sehr unterschiedlich und muss individuell angepasst werden.

Die Vorbereitung auf den Transfer muss neben dem chronologischen Alter viele weitere Aspekte berücksichtigen: neben der körperlichen auch die kognitive Reife, den aktuellen Gesundheitszustand, die Bereitschaft des Betroffenen aber auch der Eltern und der Behandler. Das von den Eltern unabhängige Durchführen der Therapie und das Verständnis für die Krankheitsabläufe, die Verfügbarkeit einer adäquaten Erwachsenenbetreuung und der vergleichbaren Therapiekonzepte spielen hierbei eine wichtige Rolle. Fehlende Absprachen und Vereinheitlichungen führen zu Compliance-Problemen, können im Patienten Ablehnung und Zurückhaltung wecken woraus letztendlich eine Verschlechterung im Krankheitsverlauf droht.

Mit einem Erfolg kann gerechnet werden wenn, der Transfer von Pädiatern mit einer Bereitschaft zum „Abgeben“ initiiert wird und auf der Seite der Erwachsenenmedizin auf eine Präsenz kompetenter internistischer Strukturen mit einer Bereitschaft zum „Aufnehmen“ trifft. Nicht zuletzt muss auch eine finanzielle Absicherung des Transferprozesses gewährleistet sein.

\section{INTDIS-SY-TP-4}

\section{Familienschulungen in der pädiatrischen Nephrologie}

Benz M.R. ${ }^{1}$

'Dr. von Haunersches Kinderspital, LMU München, Pädiatrische Nephrologie, München

Rationale. Chronische Nierenerkrankungen im Kindes- und Jugendalter haben Auswirkungen auf die psychosoziale Situation des Individuums, der Familie und des sozialen Umfelds. Komplexe Therapieansätze und die steigende Tendenz zur ambulanten Therapie erfordern eine Verantwortungsübernahme und Adhärenz auf Seiten der Eltern und Patienten. Moderne Begleitung chronisch Erkrankter fußt auf dem Selbstmanagementansatz („empowerment"). Familienschulungen sind pädagogische Interventionen mit psychologischen und medizinischen Inhalten für Kinder mit chronischen Erkrankungen und deren Familien.

Die Familienschulungsprogramme. In der pädiatrischen Nephrologie wurden sowohl, für die seltenen Erkrankungen nephrotisches Syndrom und die chronische Niereninsuffizienz (CNI), als auch für Kinder mit funktioneller Harninkontinenz Familienschulungen entwickelt. Das Blutdruck-Modul kann aus der Schulung CNI ausgekoppelt verwendet werden. Auch eine Familienschulung für transplantierte Patienten wurde entwickelt. Sie ist unabhängig vom transplantierten Organ einsetzbar. Organisation. Zur ambulanten Schulung werden Gruppen aus 5-7 betroffenen Kindern ähnlichen Alters sowie deren Eltern und Geschwister werden zur Familienschulung eingeladen, die über 2 Tage stattfindet. In Reha-Kliniken werden die Schulungsinhalte in den Reha-Plan integriert. Die Veranstaltungen sind mittels modularisierten Stunden- plans strukturiert. Um altershomogene Gruppen trotz der Seltenheit der Erkrankungen zusammenstellen zu können, ist oft eine regionale Zusammenarbeit aus mehreren Zentren sinnvoll.

Ausblick. Mit den Familienschulungen in der pädiatrischen Nephrologie stehen multidisziplinäre, multimodale Schulungskonzepte für Kinder und Jugendliche mit chronischen Nierenerkrankungen sowie deren Familien zur Verfügung.

\section{Interdisziplinäre Versorgung krebserkrankter Kinder}

\section{INTDIS-SY-IV-2}

Standards und Kontroversen bei der Bildgebung von Lungenmetastasen im Kindesalter

Hirsch F.W. ${ }^{1}$

'Universität Leipzig, Selbstständige Abteilung für Kinderradiologie, Leipzig

Die Aufgabe der Kinderradiologie ist es, Lungenmetastasen oder einen Lungenbefall im Rahmen von Lymphomen in der erforderlichen Genauigkeit nachzuweisen oder auszuschließen.

Falls ein GPOH-Protokoll für die Tumorentität vorliegt, kommen hierzu bisher das Röntgen in zwei Ebenen und die Thorax-CT infrage. Obwohl das CT bekanntermaßen deutlich mehr und kleinere Metastasen detektiert als die Röntgenaufnahme, gibt es inzwischen Hinweise, dass die dadurch verbesserte Metastasen-Diagnostik bei einzelnen Tumorentitäten - z. B. beim Nephroblastom - nicht zu einem verbesserten Outcome führt. Zudem ist unbedingt die erhebliche Strahlenexposition durch das CT zu berücksichtigen. Man rechnet heute mit einem induzierten Malignom auf 380 Thorax-CT-Untersuchungen.

Leider tragen die Protokolle der GPOH und andere Richtlinien an dieser Stelle dem medizinischen Fortschritt in der Lungenbildgebung kaum Rechnung. Inzwischen ist die Lungen-MRT - und an einzelnen Standorten auch das PET-MRT - eine Option, die allerdings in den Protokollen noch keinen Niederschlag gefunden hat. Die Gründe hierfür sind vielfältig und werden diskutiert. Der Einsatz des Lungen-MRT erfolgt bisher meist außerhalb der GPOH-Schemata, überwiegend zur Verlaufskontrolle von schon nachgewiesenen Lungenmetastasen.

\section{Pädiatrische Intensivmedizin: interdisziplinäre Schnittstelle in einer Kinderklinik}

\section{INTDIS-SY-PI-3}

Pädiatrische Stroke Units - die Zukunft der Schlaganfallversorgung im Kindesalter aus Sicht der Kinderneurologie und Hämostaseologie Gerstl L. ${ }^{1}$, Borggräfe I. ', Heinen F. ', Nicolai T. ${ }^{2}$, Reiter K. ${ }^{2}$, Kurnik K. ${ }^{3}$, Olivieri M. ${ }^{3}$ 'Dr. von Haunersches Kinderspital, Ludwig-Maximilians-Universität München, Abteilung für Pädiatrische Neurologie, Entwicklungsneurologie und Sozialpädiatrie, iSPZ hauner, München, ${ }^{2}$ Dr. von Haunersches Kinderspital, Ludwig-Maximilians-Universität München, Abteilung für Pädiatrische Intensivmedizin, München, ${ }^{3} \mathrm{Dr}$. von Haunersches Kinderspital, LudwigMaximilians-Universität München, Abteilung für Pädiatrische Hämostaseologie, München

Der kindliche Schlaganfall ist ein pädiatrischer Notfall mit hoher Morbidität, Mortalität und einem erheblichen Rezidivrisiko. In Deutschland sind 300-500 Kinder/Jahr davon betroffen. Aufgrund heterogener Ätiologie und Risikofaktoren sowie klinischer Präsentation ist die Diagnosestellung häufig schwierig und mit einem erheblichen Zeitverlust verbunden. Nur ein Drittel der Kinder mit Schlaganfall werden innerhalb der ersten 6 Stunden nach Symptombeginn diagnostiziert und behandelt. In über $50 \%$ der Kinder wird der Schlaganfall primär nicht als solcher erkannt. Die Akuttherapie und insbesondere der Einsatz der Lysetherapie erfordern hohe Expertise und eine kritische Risiko-Nutzen-Abwägung im Pediatric Stroke Unit Team. 
Zur Verbesserung der Versorgungssituation pädiatrischer Schlaganfallpatienten wurde bundesweit die erste interdisziplinäre Pädiatrische Stroke Unit auf der Kinderintensivstation des Dr. von Haunerschen Kinderspitals eröffnet. Ein Team aus erfahrenen Kinderneurologen, Hämostaseologen, Intensivmedizinern, Neuro- und Kinderradiologen steht durchgehend (24/7) zur Verfügung um jederzeit die optimale Diagnostik und Therapie bei Kindern mit Schlaganfall oder schlaganfallähnlichen Symptomen zu gewährleisten.

Aus der 20-jährigen Erfahrung in der Schlaganfallbehandlung bei Erwachsenen soll durch ein analoges, strukturiertes und spezialisiertes Management von Kindern mit V. a. Schlaganfall auf der Pediatric Stroke Unit eine Verbesserung von Outcome und Prognose erreicht werden. Die Pediatric Stroke UnitLMU ist ein universitäres Kooperationsprojekt mit der Neurologie/dem Schlaganfallzentrum am Campus Großhadern, dem integrierten Sozialpädiatrischen Zentrum (iSPZ hauner) und der Deutschen Schlaganfall Gesellschaft.

\section{Thermische Verletzungen im Kindesalter}

\section{INTDIS-SY-TV-2 \\ Der Stellenwert der Physiotherapie/Ergotherapie bei der Behand- lung thermischer Verletzungen im Kindesalter}

\section{Ziegenthaler $\mathrm{H}^{?}$}

'Gräfliche Kliniken, Rehabilitations-Fachklinik für Orthopädie/Traumatologie und Neurologie - Reha Zentrum für Brandverletzte, Bad Klosterlausnitz

Thermische Verletzungen rufen Beeinträchtigungen der Körperfunktionen und -strukturen, von funktionellen Fähigkeiten und sozialer Integrität hervor. Hohen Erwartungen an alltagsrelevante Funktionalität und Ästhetik der Betroffenen und deren Eltern stehen oft hypertrophe Narbenbildungen mit Kontrakturen oder Fehlstellungen entgegen.

Beim intensiven Einsatz qualifizierter funktionsorientierter Interventionen in der Rehabilitationsphase müssen Besonderheiten vorausgegangener plastisch-chirurgischer Maßnahmen Beachtung finden. Das Ziel, wieder Selbständigkeit im Alltag und unbeschwerte soziale Integrität zu ermöglichen, Perspektiven für spielerischen und schulischen Wiedereinstieg zu eröffnen, fordert allen Therapeuten Einfallsreichtum bei der Methodenauswahl, psychoemotionales Einfühlungsvermögen auch im Umgang mit den Eltern ab.

In der Praxis ergänzen sich motorisch-funktionelle und sensorischperzeptive Behandlungen mit jeweils spielerischem Ansatz. Funktionelle Zielsetzungen der Kontrakturbehandlungen werden in therapiefreien Zeiten durch funktionsverbessernde Schienen umgesetzt. Im Haushalts- und Sozialtraining erfährt die Gesamtheit der körperlichen und seelischen Betroffenheit Berücksichtigung.

Anhand von ausgewählten Fallbeispielen aus dem RehaZentrum für Brandverletzte werden foto- und videodokumentierten die therapeutische Vielfalt und Ergebnisse der therapeutischen Interventionen dargestellt.

Nur ein komplexes sowie individuelles und zugleich vielseitiges Rehabilitationskonzept kann den spezifischen Ansprüchen des brandverletzten Kindes gerecht werden. Es benötigen ebenso wie die Eltern bei den ersten Schritten im „neuen Leben“ Begleitung, die sich durch Sachkompetenz, Professionalität, Erfahrung, zielgerichtetes Handeln und Einfühlungsvermögen auszeichnet.

\section{INTDIS-SY-TV-3}

Sekundär-chirurgische Behandlungsstrategien bei thermischen Verletzungen im Kindesalter

Königs $I^{1}$

'AKK Altonaer Kinderkrankenhaus gGmbH, Abteilung für Kinderchirurgie, Sektion für Brandverletzungen, plastische und rekonstruktive Chirurgie im Kindesalter, Hamburg

Eine qualitativ hochwertige Behandlung von Kindern mit thermischen Verletzungen besteht nicht nur aus der Akutbehandlung. Um bestmögliche Behandlungsergebnisse $\mathrm{zu}$ erzielen, muss eine kontinuierliche Nachbetreuung der Patienten über Jahre erfolgen, um die Dynamik der Narbenentwicklung $\mathrm{zu}$ beobachten und auch gegebenenfalls weitere notwendige Therapiemaßnahmen oder Korrektureingriffe zu initiieren. Dazu werden die Kinder langfristig in einer speziellen Verbrennungssprechstunde begleitet, die in enger Zusammenarbeit mit orthopädischen Fachwerkstätten und Physiotherapeuten stattfinden sollte. Diese Nachbetreuung sollte in dem Behandlungskonzept bei Kindern mit thermischen Verletzungen einen mindestens ebenso hohen Stellenwert einnehmen wie die Akuttherapie. Die konservative und insbesondere operative Betreuung dieser Kinder sollte erfahrenen Behandlern mit ausreichend großer Expertise vorbehalten bleiben.

Sekundäre chirurgische Korrekturen können nach Ausschöpfen der konventionellen Maßnahmen durch funktionelle aber auch ästhetische Einschränkungen notwendig werden. Indikationen sollten jedoch insbesondere bei ästhetischen Eingriffen im Kindesalter streng gestellt werden.

Die verschiedenen operativen Strategien werden ausführlich beleuchtet. Dazu gehören kleinere Eingriffe wie die Laserchirurgie/-therapie, Needling, oder lokale Narbenplastiken (Z-Plastiken, Jumping-man-Plastiken etc.) wie auch aufwendigere Verfahren wie Narbenrelease/-exzisionen mit Vollhaut-Transplantationen oder Dermisersatzverfahren. Ebenso werden aber auch die seltener verwendeten Behandlungsmethoden wie Expanderverfahren (konventionell und osmotisch), „dermal overgrafting" und größere Rekonstruktionen mit Dermisersatzstoffen bezüglich Indikation und zu erreichender Ergebnisse dargestellt.

Ziel ist es, einen Überblick über sinnvolle chirurgische Möglichkeiten zu geben, sollte mittels konservativen Maßnahmen ein zufriedenstellendes funktionelles oder ästhetisches Ergebnis nach thermischen Verletzungen nicht erreicht werden.

\section{Chronisch-entzündliche Darmerkrankungen (CED)}

\section{INTDIS-SY-CE-1}

Was wir von der molekularen Epidemiologie über die Pathophysiologie chronisch-entzündlicher Darmerkrankungen gelernt haben

Posovszky C. $^{1}$

'Universitätsklinikum Ulm, Klinik für Kinder- und Jugendmedizin, Ulm

Chronisch-entzündliche Darmerkrankungen (CED), wie z. B. Morbus Crohn, Colitis ulcerosa und Colitis indeterminata, treten häufig bereits im Kindesalter auf. Die Inzidenz für den Morbus Crohn liegt bei 2-8 und für Colitis ulcerosa bei 4-10×105 Einwohner, bei einer Prävalenz von 50-100 bzw. 40-115 ×105 Einwohner.

Es handelt sich um Erkrankungen mit multifaktorieller Genese. Neben Umwelteinflüssen durch Hygienestandards und Infektionen spielen genetische Faktoren eine Rolle. Eine familiäre Häufung von CED findet sich bei ca. 10\% der Erkrankten und bei 50-60\% eineiiger Zwillinge. Durch genomweite „Kopplungsanalysen“ erkrankter Geschwisterpaare konnten mehrere Genregionen identifiziert werden. 2001 wurden erstmals in der Kopplungsregion 16q12 drei Genvarianten von NOD2 beschrieben, die ein Erkrankungsrisiko für M. Crohn darstellen. Die Reaktion auf Umwelteinflüsse und die immunologische Antwort wird genetisch determiniert. 
Die Darmschleimhaut bildet eine Epithelbarriere und sezerniert antimikrobielle Peptide (z. B. Defensin), IgA und Mucus zum Schutz gegenüber der Umwelt. Störungen der chemischen, immunologischen und mechanischen Abwehrmechanismen führen zu CED. Bei Erkrankten besteht eine Barrierestörung der Darmschleimhaut, Bakterien können dann ungehindert in die Darmwand eintreten und Entzündungsreaktionen auslösen. Ein Ungleichgewicht von entzündungsfördernden und entzündungshemmenden Botenstoffen spielt bei diesen Erkrankungen eine zentrale Rolle in der Pathogenese, die auch zur Entwicklung gezielter immunologischer Behandlungsstrategien geführt hat (z. B. antiTNF-a-Antikörper).

Die aktuellen Fortschritte der Ursachen- und Therapieforschung zu CED basieren im Wesentlichen auf der molekularen Epidemiologie und den Ergebnissen therapeutischer Experimente an Patienten.

\section{Impfungen bei Patienten mit Immuninsuffizienz}

\section{INTDIS-SY-II-1 \\ Impfungen bei Patienten mit Immuninsuffizienz}

von Bernuth H. ${ }^{1,2}$

'Charité - Universitätsmedizin Berlin, Kinderklinik m. S. Pneumologie und Immunologie, Berlin, ${ }^{2}$ Labor Berlin Charité Vivantes GmbH, Fachbereich Immunologie, Berlin

Impfungen sind segensreich. Mittels weltweiter Impfanstrengungen gelang die Ausrottung der Pockenviren. Die Ausrottung der Masernviren und Polioviren sind von der World Health Organisation (WHO) verfolgte Ziele, die im Falle der Poli „nur“ an der mangelnden Logistik und Infrastruktur in wenigen Ländern (insbesondere Afghanistan, Pakistan, Nigeria, Somalia) und im Falle der Masern auch an der ungenügenden Durchimpfungsrate in vielen Ländern Europas scheitern. Eine der häufiger angeführten Kontraindikationen gegen Impfungen nach den Empfehlungen der Ständigen Impfkommission (STIKO) in Deutschland ist die Angst vor Impfungen bei zu diesem Zeitpunkt noch unerkannten angeborenen Immundefekten. Für die Impfung von Totimpfstoffen ist diese Angst unbegründet, da diese auch beim immuninsuffizienten Impfling keine Erkrankung hervorrufen können. Zur Ausbildung einer ausreichenden Impfantwort mit langfristig schützenden Antikörpertitern und somit auch zur Kontrolle von attenuierten Lebendimpfstoffen ist das Zusammenspiel Antigen-präsentierender Zellen (Makrophagen/Dendritische Zellen) mit T-Zellen und B-Zellen notwendig. Daraus leitet sich ab, dass Lebendimpfstoffe vor allem bei Störungen in diesem Bereich des Immunsystems eine Gefährdung für den Impfling darstellen können. Im Zweifelsfall empfiehlt die STIKO die Rücksprache mit einem pädiatrischen Immunologen/Infektiologen. Der einfachste und wirksamste Schutz für Patienten mit Immundefekt kann über eine konsequente Impfung aller gesunder Umgebungspersonen („Herdenimmunität“) erreicht werden.

\section{INTDIS-SY-II-2 \\ Impfungen bei Patienten mit chronisch-rheumatischen Erkrankun- gen}

Borte M. ${ }^{\prime}$

'Klinik für Kinder- und Jugendmedizin am St. Georg Leipzig, Fachbereich für Pädiatrische Rheumatologie, Immunologie und Infektiologie, Leipzig

Kinder und Jugendliche mit chronisch-rheumatischen Erkrankungen tragen ein deutlich höheres Infektionsrisiko als Gesunde. So beträgt die Rate schwerer bakterieller Infektionen bei Kindern mit juveniler idiopathischer Arthritis (JIA) 2,4 pro 100 Patientenjahre und liegt damit etwa 1,25-fach über der eines Kontrollkollektivs. Bei einer Behandlung mit oralen Glukokortikoiden erhöht sich die Rate auf 9,8 pro $100 \mathrm{~Pa}-$ tientenjahre. Wirkungen und Nebenwirkungen von Impfungen bei
Kindern, Jugendlichen und Erwachsenen mit rheumatischen Erkrankungen waren bis vor kurzem kaum untersucht. Inzwischen liegen aber gute Daten zur Sicherheit und Wirksamkeit der meisten im Impfkalender enthaltenen Standardimpfungen für Kinder und Erwachsene mit Rheuma vor.

Generell sind Kinder, Jugendliche und Erwachsene mit rheumatischen Erkrankungen entsprechend der Empfehlungen der Ständigen Impfkommission (STIKO) am Robert-Koch-Institut Berlin zu impfen. Bei Rheumakranken festgestellte Impflücken, insbesondere für Standardimpfungen mit Tot-Impfstoffen (z. B. gegen Tetanus, Diphtherie, Pertussis und Poliomyelitis) sollte es nicht geben. Indiziert ist bei Rheumapatienten auch die saisonale Influenza-Schutzimpfung. Auch unter einer standardisierten, niedrig dosierten Immunsuppression sind Impfungen möglich. Der Zeitpunkt der anstehenden Impfung ist in Abhängigkeit von der Krankheitsaktivität und Therapie sorgfältig zu wählen und ggf. der Impferfolg zu überprüfen.

Rheumapatienten ist eine besondere intrinsische und therapiebedingte Infektionsanfälligkeit eigen. Diese trägt wesentlich zur Morbidität und Mortalität bei. Bei einem hohen Anteil der Infektionen handelt es sich aber um impfpräventable Erkrankungen.

\section{Innovationen in der Rehabilitation}

\section{INTDIS-SY-IR-3 Neue Konzepte in der Ataxiebehandlung}

Synofzik M. ${ }^{1}, \operatorname{llg} W^{2}{ }^{2}$

'Zentrum für Neurologie, Tübingen, ${ }^{2}$ Hertie-Institut für Klinische Hirnforschung, Tübingen

Degenerative und neurometabolische Ataxien stellen eine große therapeutische Herausforderung dar. Für den überwiegenden Teil gibt es bislang keine effektiven medikamentösen Behandlungsansätze. Nun konnten wir zeigen, dass rehabilitative Ansätze nachweislich zu einer Verbesserung ataxie-spezifischer Dysfunktionen mit hoher Alltagsrelevanz führen.

Im ersten Schritt konnten wir nachweisen, dass physiotherapeutische Übungen mit Schwerpunkt auf Koordination zu einer Besserung degenerativer Ataxie führen. Diese Übungen bringen jedoch insbesondere bei Kindern und Jugendlichen mit Ataxie mehrere Nachteile mit sich. Insbesondere lässt bei betroffenen Kindern und Jugendlichen oftmals die Motivation nach. Wir haben nun in einem zweiten Schritt ein Trainingsprogramm entwickelt, das auf kostengünstige, kommerziell erhältliche Ganzkörper-kontrollierte Videospiele („Exergames“) zurückgreift. Dieses Training führte zu einer alltagsrelevanten Verbesserung der Ataxie und zum (Wieder-)Erleben von motorischen Erfolgserlebnissen. In einem dritten Schritt wurde exemplarisch überprüft, ob diese Trainingsmethode auch bei bereits rollstuhlpflichtigen Kindern und Jugendlichen mit fortgeschrittener multisystemischer Neurodegeneration effektiv ist. Auch hier ließen sich Verbesserungen in der Rumpfund Standmotorik erreichen.

Zusammengefasst bieten „Exergames“ eine attraktive neue Trainingsmethode: Sie ist kostengünstig, kann von Kindern und Jugendlichen selbstständig zu Hause durchgeführt werden, hält die Motivation und Freude an regelmäßigem Training aufrecht, und vermittelt ein Erleben der „Selbstwirksamkeit“ in dem Umgang mit der Erkrankung. Sie ist wahrscheinlich nicht nur für Kinder mit degenerativer Ataxie geeignet, sondern auch für Kinder mit anderweitigen Bewegungsstörungen. 


\section{Chronische Schmerzen bei Kindern}

\section{INTDIS-SY-CS-1 \\ Vom CRPS bis zur Migräne, von selten bis täglich: Diagnose und Therapie chronischer Schmerzen in Praxis und Klinik}

\section{Zernikow B. ${ }^{1}$}

'Deutsches Kinderschmerzzentrum, Vestische Kinder- und Jugendklinik, Datteln - Universität Witten/Herdecke, Datteln

Chronische muskuloskeletale Schmerzen werden sowohl primär als auch sekundär, bei einer großen Zahl angeborener und erworbener Erkrankungen, beobachtet. Eine besondere diagnostische und therapeutische Herausforderung ist das Complex Regional Pain Syndrom (CRPS, früher Morbus Sudeck). Bei chronischen Kopfschmerzen stehen die Unterscheidung von Spannungskopfschmerzen (SK) und Migräne (M), die gezielte medikamentöse Therapie der Migräneattacke, die Vermeidung von Analgetika bei SK und einfache multimodale Ansätze für die Kinder- und Jugendarztarztpraxis im Vordergrund. Funktionelle gastrointestinale Störungen sind die führende Ursache chronischer Bauchschmerzen und sollten frühzeitig einem multimodalen Behandlungsansatz zugeführt werden. Deren Prinzipien und wichtigsten Behandlungsmodule werden systematisch dargestellt. Wesentliche Behandlungsziele sind die Steigerung der körperlichen Leistungsfähigkeit, Verminderung der Schmerzwahrnehmung, Förderung von Kontroll- und Selbstwirksamkeits-Erfahrungen sowie die Wiederaufnahme normaler Alltagsstrukturen und sozialer Kontakte.

\section{INTDIS-SY-CS-2 \\ Kindern und Jugendlichen Schmerzen erklären: die altersgerechte (Video-)Edukation}

\section{Wager J.' \\ 'Vestische Kinder- und Jugendklinik Datteln, Deutsches Kinderschmerz- zentrum, Datteln}

Bei chronischen Schmerzen stellt die Edukation einen elementaren Bestandteil der Therapie dar. Die Edukation ist ein wichtiges Instrument, um ein bio-psycho-soziales Schmerzverständnis zu vermitteln. Nur wenn die pädiatrischen Schmerzpatienten und ihre Eltern ein solches Verständnis bezüglich des Schmerzproblems haben, ist es möglich, unterschiedliche therapeutische Ansätze zu realisieren. Edukation findet im Idealfall in einem intensiven Patient-Therapeuten-Gespräch statt. Jedoch stehen die wenigen zeitlichen Ressourcen oftmals im Widerspruch zu einem intensiven Gespräch. Ein vom Deutschen Kinderschmerzzentrum entwickeltes Edukationsvideo „Den Schmerz verstehen - und was zu tun ist in 10 Minuten "kann (unterstützend) eingesetzt werden, um Kindern und Jugendlichen mit chronischen Schmerzen das bio-psycho-soziale Schmerzmodell näher zu bringen. In ihrem Vortrag wird Dr. Wager auf wichtige Bestandteile der Edukation bei chronischen Schmerzen eingehen. Darüber hinaus wird sie Informationen zur Anwendung der Videoedukation geben.

Link zum Video: http://www.youtube.com/watch?v=KpJfixYgBrw

\section{Antibiotika Stewardship}

INTDIS-SY-AS-4

Kosten und Einsparmöglichkeiten bei adäquatem Antibiotikaeinsatz

Beyersdorff A. ${ }^{\prime}$, Bruns R. ${ }^{1}$, Simon A. ${ }^{2}$

'Zentrum für Kinder- und Jugendmedizin Universitätsmedizin, Greifswald, ${ }^{2}$ Klinik für Pädiatrische Onkologie und Hämatologie Universitätsklinikum des Saarlandes, Homburg

Einführung. Das wichtigste Ziel des rationalen und restriktiven Einsatzes von Antibiotika im Rahmen eines Antibiotic Stewardship Programms (ASP) bleibt eine erfolgreiche Therapie mit möglichst wenig unerwünschten Wirkungen. Ein ASP soll sich positiv auf das Resistenzprofil auswirken. Außerdem werden im Verlauf eines ASP Kosteneinsparungen erwartet.

Methode. Literaturrecherche über PubMed unter den Suchkriterien "costs + hospital + antibiotics, antibiotic resistance + costs, antimicrobial stewardship program + costs", CDC Internetsuche.

Ergebnisse. Durch ein ASP kann der Verbrauch von Antibiotika signifikant gesenkt werden, zum einen durch den gezielteren Einsatz, eine verkürzte Behandlungsdauer, eine frühe orale Sequenztherapie und den selteneren Einsatz kostenintensiver Reservetherapeutika, was ebenso zu einer Kostenersparnis führt. Gut geführte ASP verbessern das Behandlungsergebnis und führen nicht zu einer Gefährdung der Patienten. Lt. Recherche werden z. B. $13 \%$ der Infektionen durch resistente Erreger hervorgerufen (Roberts 2009) oder 29\% der nosokomialen Infektionen durch resistente bzw.16\% durch multiresistente gram negative Erreger verursacht (Mauldin 2009). An einigen Orten waren Einsparungen von bis zu 80\% durch Antibiotic Stewardship Programme möglich (CDC 2013), oder es konnte der Antibiotikaverbrauch um 35\% gesenkt werden und damit die Kosten für Antibiotika um 53\%. Zum einen geschah dies durch 35\% weniger Verordnungen und zum anderen durch 13\% niedrigeren Einsatz von Breitspektrumantibiotika (Rüttimann 2004).

Schlussfolgerung. Nach den Ergebnissen dieser Literaturanalyse führen ASP zu einer signifikanten Kostenersparnis und nicht zu einer Gefährdung der Patienten durch weniger Therapie. Der Einfluss auf endemische Resistenzprofile muss weiter untersucht werden.

\section{Schütteltrauma-Syndrom - Konzepte und Kontroversen}

\section{INTDIS-SY-SC-1}

Epidemiologie und Klinik des STS

\section{Herrmann B.}

'Klinikum Kassel, Klinik für Kinder- und Jugendmedizin, Kassel

Das Schütteltrauma-Syndrom ist die Misshandlungsform mit der höchsten Letalität und schwerwiegendsten Morbidität. Sowohl hinsichtlich der Erkennung, akutmedizinischen Betreuung, Nachsorge als auch der sich daraus ergebenden forensischen Implikationen ist die Kenntnis der definierenden Elemente und der Pathophysiologie von großer Bedeutung. Dies gilt insbesondere für die zeitliche Einordnung der Symptome und die neurologische Prognose, für die die definierenden klinischen Charakteristika der subduralen und retinalen Blutungen von geringer Bedeutung sind. Die Daten zur Inzidenz sind begrenzt und wenige Studien wurden prospektiv angelegt. Die hierzu vorliegenden Daten einschließlich der deutschen ESPED Erhebung 2006-2009 werden vorgestellt. 


\section{INTDIS-SY-SC-3}

\section{Schütteltrauma-Syndrom: diagnostische Probleme, Umstrittenes,} Kontroversen

\section{Sperhake J.P. ${ }^{1}$ \\ 'Universitätsklinikum Hamburg Eppendorf, Institut für Rechtsmedizin, Hamburg}

Das Konzept des Schütteltraumas ist nicht unumstritten. Die wissenschaftliche Debatte wurde auch in die Gerichtssäle getragen und hat zu einer Verunsicherung von Laien und Juristen geführt. Ein Teil der Probleme resultiert aus der prinzipiellen Unmöglichkeit, auf diesem speziellen Gebiet evidenzbasierte Forschung zu betreiben. Ausgewählte Kontroversen werden dargestellt: Kann man die Diagnose Schütteltrauma („shaken baby syndrome“, SBS) überhaupt stellen? Entsteht die Trias subdurales Hämatom, Enzephalopathie und retinale Blutung auch nichttraumatisch? Wie viel Gewalt ist notwendig, damit ein Schütteltrauma entsteht? Führen Stürze aus geringer Höhe zu tödlichen Kopfverletzungen? Gibt es ein luzides Intervall nach einem signifikanten Schütteltrauma? Welchen diagnostischen Stellenwert haben retinale Blutungen? Trotz der genannten Einschränkungen gibt es plausible Konzepte, die die Diagnose einer nichtakzidentellen Kopfverletzung („non-accidental head injury“, NAHI) untermauern. Ein multidisziplinärer Ansatz führt zu einer deutlich höheren Sicherheit bei der Diagnosestellung.

\section{INTDIS-SY-SC-4}

Prognose und neurologisches Outcome bei Schütteltrauma-Syndrom

\section{Baz Bartels M. ', Schmitz N. ', Knotz J.', Blank A.-E. ', Kieslich M. ${ }^{\text {' }}$ ${ }^{1}$ Klinik für Kinder- und Jugendmedizin, Abteilung für Neuropädiatrie, Frankfurt am Main}

Die Prognose und das Outcome nichtakzidenteller Kopfverletzungen (NAKV) in Verbindung mit dem Schütteltrauma Syndrom (STS) differiert erheblich von denen akzidenteller Kopfverletzungen (AKV). Im Vordergrund stehen dabei die neurologischen Defizite (60-70\%), die in Verbindung mit einem hohen Mortalitätsrisiko (20-25\%) zu einer Gesamtmorbidität von ca. 90\% beitragen und somit höher liegen als bei AKV. Ätiologisch ist der besondere Verletzungsmechanismus des STS zu beleuchten, der über massive auf den Schädel einwirkende Rotationskräfte zu großflächigen subduralen Blutungen, diffusen retinalen Einblutungen und Scherverletzungen des Hirnparenchyms führt. Erschwerend erfolgt die Vorstellung der Patienten meist erst verzögert und nach wiederholter Traumatisierung, sodass die direkt mit einer schlechteren Prognose korrelierenden Faktoren, wie der Reanimationsbedarf, das gemessene Basendefizit als Hypoxie- oder das erhöhte Liquor-Cytochrom C als Apoptosekorrelat, bereits weit fortgeschritten sind. Jedoch auch das Ausmaß der Rotationsverletzungen im Bereich des zervikomedullären Übergangs und den angrenzenden hirnstammnahen Strukturen haben, messbar mittels DWI-MRT-Sequenzen, als Zeichen der intrazellulären Ödembildung, einen prognostischen Charakter. Der Verlust zerebraler Autoregulationsmechanismen, der Untergang der Blut- Hirnschrankenfunktion, die diffuse Einblutung oder teilweise Ablösung größerer retinaler Areale, die Unterbrechung intrazerebraler ionischer Homöostase, sowie der Untergang großvolumiger, nicht auf Gefäßversorgungsgebiete begrenzter Zellverbände, führt, trotz frühzeitiger dekomprimierender Kraniektomie, häufig zu schweren zerebralen Anfallsleiden, partieller Erblindung, motorischen und kognitiven Defiziten, dauerhaft vegetativen Zuständen oder gar zum Tode.

\section{Schwerstkranke Kinder und ihre Familien - Therapieentscheidungen und Palliativversorgung}

\author{
INTDIS-SY-SK-1 \\ Die besonderen Bedürfnisse von Kindern und Jugendlichen in der \\ Palliativmedizin
}

Führer M.', Groh G.', Riester M.', Borasio G.D. ${ }^{1}$

'Dr. von Haunersches Kinderspital, Ludwig-Maximilians-Universität München, Koordinationsstelle Kinderpalliativmedizin, München

Fragestellung. Das Gesetz zur spezialisierten ambulanten Palliativversorgung (SAPV) sieht die Berücksichtigung der besonderen Bedürfnisse von Kinder und Jugendlichen vor. Ziel der vorliegenden Studien ist es, Unterschiede zwischen Erwachsenen und Kindern zu erfassen und die besonderen Versorgungsbedürfnisse anhand der Erkrankungsgruppen nach ACT zu beschreiben.

Methode. Es handelt sich um eine prospektive, vergleichende Untersuchung an Patienten des Kinder- und Erwachsenen-SAPV-Teams eines Universitätsklinikums, sowie um die retrospektive Analyse der Daten der pädiatrischen Patienten.

Ergebnisse. Zwischen 4/11 und 6/12 wurde 6o erwachsene (Median: 67,5 Jahre) und 40 pädiatrische Patienten (Median: 6 Jahre) und ihre pflegenden Angehörigen befragt. Die Daten von 123 konsekutiven pädiatrischen Pat. wurden analysiert. Bei den pädiatrischen Pat. überwogen die nicht-onkologischen Erkrankungen ( 25 vs. $87 \%$; $<<0,001$ ). In beiden Gruppen berichteten die Angehörigen über eine signifikante Verbesserung ihrer eigenen QoL und der QoL der Patienten durch die SAPV. Die psychische Belastung der Angehörigen verringerte sich signifikant. In der Analyse der Daten der pädiatrischen Pat. zeigte sich eine kürzere Betreuungsdauer bei höherer Betreuungsintensität in der ACT-Gruppe 1 (Erkrankungen mit kurativer Therapiemöglichkeit, z. B. Leukämie, Organversagen) im Vergleich zu den ACT-Gruppen 3 und 4 (fortschreitende Erkrankungen; Zustand nach schwerer neurologischer Schädigung).

Schlussfolgerung. SAPV kann bei Erwachsenen und Kindern die QoL der Patienten und der pflegenden Angehörigen verbessern und die Belastung verringern. In der pädiatrischen Stichprobe überwogen die nichtonkologischen Erkrankungen. Die verschiedenen ACT-Gruppen unterscheiden sich signifikant hinsichtlich Versorgungsdauer und -intensität.

\section{INTDIS-SY-SK-2}

Kinder- und Jugendhospizarbeit. Unterstützung und Begleitung für lebensverkürzend erkrankte Kinder und ihre Familien

\section{Jennessen $S^{1}$}

'Universität Koblenz-Landau, FB5, Institut für Sonderpädagogik, Landau

Mit welchen Angeboten unterstützt die Kinder- und Jugendhospizarbeit lebensverkürzend erkrankte Kinder und Jugendliche und ihre Familien? Trifft sie die Bedarfe und Bedürfnisse progredient erkrankter Kinder und Jugendlicher? Unterstützt sie deren Eltern und Geschwister genau da, wo sie Begleitung, Unterstützung, Hilfe und Austausch wünschen und brauchen? Und nicht zuletzt: Wie soll es weiter gehen - an welchen Stellen gibt es Entwicklungs- und Optimierungsbedarfe? Und wie sieht Qualitätsmanagement in diesem Bereich aus? Antworten auf diese Fragen stehen im Fokus dieses Vortrags.

Kinder- und Jugendhospizarbeit als spezifische Form der Unterstützung von Familien mit lebensverkürzend erkrankten Kindern befindet sich in Deutschland seit 1990 in einem kontinuierlichen Aufbau- und Etablierungsprozess. Vor 24 Jahren fanden sich sechs Familien progredient erkrankter Kinder zusammen, beschlossen nach englischem Vorbild spezifische Begleitungs- und Unterstützungsangebote für Familien in dieser Lebenssituation auf den Weg zu bringen und gründeten den Deutschen Kinderhospizverein e. V. Die aus dieser Idee entwickelten 
Angebote für betroffene Familien weisen mittlerweile eine große Bandbreite auf. Neben den stationären Kinder- und Jugendhospizen entwickelt sich der Bereich der ambulanten Kinderhospizdienste in den vergangenen Jahren sukzessive zu einem wichtigen Pfeiler der Begleitung von Eltern, ihren erkrankten Kindern und deren Geschwistern. Auch die Vernetzungs- und Bildungsangebote für die verschiedenen Zielgruppen, zu denen auch die in unterschiedlichen pädagogischen und pflegerischen Kontexten tätigen Fachkräfte gehören, differenzieren sich qualitativ und quantitativ weiter aus, werden öffentlichkeitswirksam angeboten und stark frequentiert.

\section{INTDIS-SY-SK-3 \\ Advance Care Planning bei Kindern mit lebensverkürzenden Er- krankungen}

Lotz J.D. ', Jox R.J. ${ }^{2}$, Borasio G.D. ${ }^{3}$, Führer M.

${ }^{1} \mathrm{Dr}$. von Haunersches Kinderspital, Kinderpalliativmedizin, München, ${ }^{2} \mathrm{n}$ stitut für Ethik, Theorie und Geschichte der Medizin, Ludwig-Maximilians Universität, München, ${ }^{3}$ Centre Hospitalier Universitaire Vaudois, Service de Soins Palliatifs, Lausanne, Schweiz

Hintergrund. Advance Care Planning (ACP) ermöglicht eine bessere Versorgung und eine selbstbestimmte Behandlung bei Einwilligungsunfähigkeit. Bei Erwachsenen wurde ACP erfolgreich umgesetzt und evaluiert. In der Pädiatrie existieren kaum Initiativen und Studien zu ACP. Konzepte aus der Erwachsenenmedizin können jedoch nicht uneingeschränkt übertragen werden aufgrund wichtiger Unterschiede in medizinischer, psychosozialer und juristischer Hinsicht. Ziel des Projekts ist eine Bedarfsanalyse unter Fachkräften und Eltern in Bezug auf ACP.

Methodik. Es wurden semistrukturierte Interviews mit Fachexperten $(n=17)$ in der Versorgung schwerkranker Kinder sowie betroffenen Eltern $(n=10)$ durchgeführt. Wichtige Merkmale der Teilnehmer wurden variiert: Berufsgruppe/Fachbereich, Versorgungssetting, Diagnose und Alter des Kindes. Die Interviews wurden inhaltsanalytisch ausgewertet. Ergebnisse. Fachkräfte und Eltern bewerten ACP als sinnvolles Instrument. Wichtige Elemente sind: ein kontinuierlicher Fallverantwortlicher, umfassende Aufklärung, Notfall-, Betreuungs- und Alltagsplanung. Für die Fachkräfte steht im Vordergrund: Sicherheit und Entlastung von der Verantwortung durch schriftliche Dokumentationen. Unsicherheiten in Bezug auf die Gültigkeit schriftlicher Dokumente führen jedoch zu Schwierigkeiten bei ihrer Umsetzung. Dies betrifft v. a. psychosoziale Fachkräften und Notärzte. Für die Eltern steht im Vordergrund: Information, Entlastung und Sicherheit durch Gespräche. Schwierigkeiten sind: unsichere Prognosen, Vermeidung belastender Gespräche durch Eltern und Ärzte.

Diskussion. Es besteht ein Bedarf an ACP. Die Bedürfnisse von Fachkräften und Eltern unterscheiden sich. Die Ergebnisse dieser Studie liefern wichtige Anhaltspunkte für die Entwicklung zukünftiger ACPProgramme.

\section{INTDIS-SY-SK-6 \\ Therapieentscheidungen am Lebensende in pädiatrischer Intensiv- medizin und Onkologie in Deutschland}

\section{Classen C.F.', Nennhaus M. ${ }^{1}$ \\ 'Universitäts-Kinder- und Jugendklinik, Rostock}

Hintergrund. Entscheidungen am Lebensende sind immer eine ethische Herausforderung für das medizinische Team, besonders wenn es um Kinder und Jugendliche geht. Wir untersuchten, wie an deutschen Kliniken die Entscheidungsfindungsprozesse ablaufen und wie sich diese auf das Team auswirken; u. a., wie sehr diese Entscheidungen die Teilnehmer belasten, und ob es dabei zu Konflikten kommt.

Methodik. Ein eigens entwickelter anonymisierter Fragebogen wurde an 297 Personen (Ärzte, Pflegepersonal, Therapeuten und Psychologen pädiatrisch onkologischer und intensivmedizinischer Stationen) versendet, an Kliniken, die sich zur Teilnahme an der Untersuchung bereit erklärt hatten. Die Daten wurden mit IBM SPSS Statistics 20 deskriptiv ausgewertet.

Ergebnisse. Von den 297 versendeten Fragebögen erhielten wir $77 \mathrm{zu}$ rück (25,92\% Rücklaufquote), davon 53 aus der Onkologie. Zum Ablauf der Entscheidungsfindung wurde mit Abstand am häufigsten (80,52\%) geantwortet, dass sich das Klinikteam erst intern eine Haltung bildet und dann mit dieser an die Eltern und den/die Patienten/-in heran tritt. Bei 35,6\% kam eine Zusammenarbeit mit einem Klinischen Ethikkommittee gelegentlich oder oft vor. Wenn im Entscheidungsfindungsgespräch keine eindeutige Entscheidung getroffen werden konnte, empfanden es 92,8\% der Teilnehmer als belastend. Auf die Frage, wie häufig Konflikte bei der Entscheidungsfindung das Team belasten, antworteten $22,37 \%$ mit oft und $11,84 \%$ mit immer. Kommt es zu Konflikten, spielen sich diese auf allen Ebenen ab: zwischen Berufsgruppen, zwischen Hierarchieebenen und zwischen Einzelpersonen.

Schlussfolgerung. Therapieentscheidungen am Lebensende in der Pädiatrie sind belastend für alle daran Beteiligten und können auch zu Konflikten im medizinischen Team führen.

\section{Enzephalitis - akute Enzephalopathie}

\section{INTDIS-SY-EA-1 Infektiöse Enzephalitis}

Häusler M. ${ }^{?}$

'Klinik für Kinder- und Jugendmedizin Uniklinik RWTH Aachen, Sektion Neuropädiatrie und Sozialpädiatrie, Aachen

Der menschliche Organismus lebt in ständiger Auseinandersetzung mit Mikroorganismen, die ihn entweder dauerhaft von außen (Mikrobiom) und innen (latente Viren) besiedeln oder akut befallen können. Reaktivierung und Erstkontakt können zur Infektion des ZNS führen. Die resultierende Schädigung des ZNS beruht auf vielfältigen Mechanismen: direkter Infektion von Zellen des ZNS, Toxinen, Verschlüssen hirnversorgender Gefäße, der Entzündungsantwort auf die Infektion (TNF- $\alpha$ ), Infekt-getriggerter Autoimmunität (anti-NMDA-RezeptorAutoantikörper) und metabolen Störungen (oxidativer Stress). Als schwerstes klinisches Bild resultiert die infektiöse Enzephalitis.

Im Rahmen einer konnatalen Infektion (TORCH) ist sie Begleiterkrankung einer systemischen Erkrankung, bei älteren immunkompetenten Kindern dominiert der zerebrale Befall (z. B. Herpes-Simplex-Virus Enzephaltis), bei immundefizienten Patienten ist das Erregerspektrum größer und schließt auch opportunistische Erreger, wie Pilze, ein. Frühes Erkrankungsalter prädisponiert zudem zu chronischen Infektionen (neonatal: TORCH. Säuglingsalter: SSPE). Diagnostisch ist der Direktnachweis mittels Kultur bzw. PCR Goldstandard. Serologische Verfahren werden erst verzögert positiv und sind insbesondere bei Immunsuppression unzuverlässig.

Antibakterielle, antivirale und antimykotische Therapien sind oft nur eingeschränkt wirksam und müssen insbesondere bei Neugeborenen und bei Immunsuppression über Monate bis Jahre durchgeführt werden. Die Wirksamkeit einer therapeutischen Immunsuppression zur Reduktion eines begleitenden immunvermittelten Schadens wird intensiv untersucht. Sie ist bisher nur für wenige Krankheitsbilder gesichert. Wichtigste Maßnahme zur Vermeidung zerebraler Infektionen sind Impfungen. 
INTDIS-SY-EA-2

FIRES, NORSE, AERRPS: epileptische Enzephalopathien mit enzephalitischer Symptomatik

\section{van Baalen A. ${ }^{1}$ \\ ${ }^{1}$ Klinik für Neuropädiatrie, Universitätsklinikum Schleswig-Holstein, Cam- pus Kiel, Kiel}

Hintergrund. Wenn wegen Infektzeichen bei Anfällen oder Status epilepticus eine infektiöse Enzephalitis vermutet wird, sich aber nicht bestätigen lässt, dann folgen immuntherapeutische Versuche und neuerdings die Suche nach neuronalen Antikörpern, da die Beschreibung der Autoimmunenzephalitis zum Paradigmenwechsel in der Diagnostik geführt hat.

Material und Methode. Aktuelle Übersicht über mögliche Ursachen sowie diagnostische und therapeutische Optionen dieser schweren refraktären Enzephalopathie (FIRES), die mehrere Namen hat.

Ergebnisse. Pleozytose und symmetrische extra-/limbische MRT-Veränderungen (z. B. der Basalganglien) sind unspezifisch und können Folge der hohen und langanhaltenden Anfallsaktivität, immun-vermittelt oder metabolisch bedingt sein. Der initiale klinische Verlauf und Autoantikörper (z. B. gegen TPO oder GluR) weisen auf eine immunvermittelte Ursache hin, die durch die Resistenz gegen First-line-Immuntherapie (Steroide, IgG, Plasmapherese) nicht ausgeschlossen ist. Genetische Testergebnisse (u. a. Exom, mtDNA) sind bis dato negativ.

Diskussion. Am wahrscheinlichsten handelt es sich um eine immunvermittelte epileptische Enzephalopathie, so dass in der akuten Phase nicht nur im Serum sondern auch im Liquor nach bisher unbekannten neuronalen Antikörpern gesucht und bei negativem Ergebnis eines kommerziellen Labors die Suche in einem Forschungslabor fortgeführt werden sollte. Außerdem ist eine aggressivere Immuntherapie (Cyclophosphamid und Rituximab) zu diskutieren.

Schlussfolgerung. In der akuten Phase sollte intensiv nach neuen Antikörpern im Liquor gesucht und eine aggressivere Immuntherapie versucht werden. Eine internationale webbasierte klinische Datenbank ist dringend erforderlich um den kompletten Phänotyp und die optimale Therapie zu erfassen.

\section{INTDIS-SY-EA-3 \\ Akute metabolische Enzephalopathien}

Husain R.A. ${ }^{?}$

'Universitätsklinikum Jena, Neuropädiatrie, Stoffwechselzentrum Thüringen, Jena

Akute Hirnfunktionsstörungen sind ein Leitsymptom bei Stoffwechsel-bedingten Entgleisungen, welche auf angeborenen oder erworbenen Störungen beruhen. Dieser Beitrag gibt einen Überblick über diesbezügliche Ursachen, Symptome, Diagnostik und Therapie. Metabolische Enzephalopathien können sich in jedem Lebensalter manifestieren. Das klinische Bild ist oft unspezifisch und durch progrediente oder fluktuierende Bewusstseinsstörungen sowie zerebrale Anfälle gekennzeichnet. Beim Neugeborenen können Trinkschwäche und Erbrechen Ausdruck einer Enzephalopathie sein. Ferner können akute Bewegungsstörungen, Schlaganfall-ähnliche Episoden und psychiatrische Symptome hinweisgebend sein. Begleitfaktoren wie Gedeih- und Entwicklungsstörungen, im Akutfall Organversagen sowie Auslöser können wegweisend sein. Da oft eine erhebliche Morbidität und Mortalität besteht und in Einzelfällen spezifische Therapien existieren, ist eine rasche Verdachts- und Diagnosestellung essentiell. Deshalb müssen angeborene Stoffwechselerkrankungen früh bei obigen Symptomen und Sepsis-/Intoxikationsartigen Erkrankungen bedacht und probatorisch therapiert werden. Für die Differenzialdiagnose wichtige Laborparameter sind Blutzucker, Elektrolyte, Blutgase, Laktat, Ammoniak und Ketone. Weiterführende Spezialdiagnostik in Blut, Urin und Liquor kann insbesondere in der Entgleisungssituation entscheidende diagnostische Hinweise geben. Die MRT-Bildgebung des ZNS ist oft hilfreich und sollte nach Möglichkeit durch eine MR-Spektroskopie ergänzt werden. Schwerpunktmäßig wird auf behandelbare Erkrankungen wie z. B. Hypoglykämie-verursachende Stoffwechselstörungen, Harnstoffzyklusdefekte, Organoazidurien, Neurotransmitter-Störungen, Vitamin-abhängige (epileptische) Enzephalopathien und GLUT1-Mangel eingegangen. 